\title{
Association between both Self-Reported and Directly Observed Physical Function and Depressive Symptoms in a US Chinese Population: Findings from the PINE Study
}

\author{
Mengting Li ${ }^{1}$, Xinqi Dong'* \\ ${ }^{1}$ PhD, Post Doctoral Research Fellow; Rush Institute for Healthy Aging; Rush University Medical Center \\ ${ }^{2}$ MD , MPH Professor of Medicine, Nursing, and Behavioral Sciences Director, Chinese Health, Aging and Policy Program Associate Director, Rush \\ Institute for Healthy Aging, Rush University Medical Center
}

Received: November 27, 2016; Accepted: December 30, 2016; Published: January 14, 2017

*Corresponding author: Xinqi Dong*, MD , MPH Professor of Medicine, Nursing, and Behavioral Sciences Director, Chinese Health, Aging and Policy Program Associate Director, Rush Institute for Healthy Aging, Rush University Medical Center; 1645 West Jackson Blvd, Suite 675, Chicago, IL60612. Tel.:+312-942-3350; Fax: +312-942-2861 E-mail: xinqi_dong@rush.edu

\begin{abstract}
Purpose: The association between physical function and depressive symptoms has been widely discussed in literature. However, the heterogeneity in measuring self-reported physical function has obfuscated the applicability of research findings while limited research investigated the association between performancebased physical function and depressive symptoms. This study aims to examine the association between both self-reported and performance-based physical function and depressive symptoms.
\end{abstract}

Methods: Data were derived from the Population Study of Chinese Elderly (PINE), a community-engaged, population-based epidemiological study of U.S. Chinese older adults aged 60 and above in the Greater Chicago area. Self-reported physical function was measured by Katz activities of daily living (ADL), Lawton instrumental activities of daily living (IADL), Index of Basic Physical Activities scale and Index of Mobility scale. Performance-based physical function was measured by Short Physical Performance Battery (SPPB). Depressive symptoms and depression were assessed by the Patient Health Questionnaire-9 (PHQ-9).

Results: Every one point higher in ADL (OR: 1.29, 1.14-1.45), IADL (OR: 1.17, 1.13-1.22), Index of Basic Physical Activities scale (OR: 1.22, 1.19-1.26), Index of Mobility scale (OR: 1.52, 1.391.66), and SPPB (OR: 1.16, 1.12-1.19) was significantly associated with higher risk of depressive symptoms. In addition, both selfreported and performance-based physical function was significantly associated with depression.

Discussion: This study initially examined the association between both self-reported and performance-based physical function and depressive symptoms and it further identified physical function impairment was not only associated with depressive symptoms, but also depression. Our study suggests that health professionals should be aware of the depressive symptoms or depression in older adults with physical function impairment.

Keywords: physical function; depressive symptoms; depression; older adults; Chinese

\section{Introduction}

Previous research reported physical function impairment had a contemporaneous and strong effect on depressive symptoms among a young and middle-aged population [1]. Understanding the relationship between physical function and depressive symptoms is particularly important for populations with high risk of physical impairment, such as older adults [2]. Physical function impairment is one of the most common features associated with aging [3]. Many prior studies confirmed that depression is more prevalent among disabled older adults [2].

The reciprocal relationship between physical function and depressive symptoms has been well documented in literature [4-6]. Existing studies indicated the effect of physical function on depressive symptoms was faster and stronger than the lagged effect of depressive symptoms on physical function [1, 2]. Some longitudinal studies found that prior levels of physical impairment predicted changes in depressive symptoms, but there was no evidence of the reverse association [7]. Depression was also more reversible than physical impairment. Thus, this study pays more attention to the association between physical function and depressive symptoms.

Prior studies mainly focused on the association between self-reported physical function and depressive symptoms, while the association between performance-based physical function and depressive symptoms has been understudied. Early studies found lower levels of self-reported physical function, including ADL, IADL, and physical functioning and mobility (PFM), were associated with higher levels of depression among South African older adults [8]. Studies also reported that increasing number of self-reported physical impairment was associated with an increased prevalence of depression in Latin American countries [9]. 


\section{PINE Study}

As limited literature documented the association between performance-based physical function and depressive symptoms, this study aims to accumulate knowledge on the relationship between both self-reported and performance-based physical function and depressive symptoms in a largely communitydwelling US Chinese old population. If the association between physical function and depressive symptoms can be supported, we'd like to go further to test whether physical function is associated with depression.

\section{Methods}

\section{Sample}

The Population Study of Chinese Elderly in Chicago (PINE) is a community-engaged, population-based epidemiological study of U.S. Chinese older adults aged 60 and above in the Greater Chicago area. The PINE study is a representative of the Chinese aging population in the Greater Chicago area with a sample size of $3,157[10,11]$. Culturally appropriate community recruitment strategies guided by community-based participatory research (CBPR) approach were used to ensure community participation [12-15]. Face-to-face home interviews were conducted by trained multicultural and multilingual interviewers. Preferred language (English or Chinese) and dialect (e.g., Cantonese, Taishanese, Mandarin, and Teochew) for participants were used during the interview. The study has been approved by the Institutional Review Board of the Rush University Medical Center [16].

\section{Measurements}

Depressive Symptoms and Depression. Depressive symptoms were measured by the Patient Health Questionnaire- 9 (PHQ-9) [17]. Participants were asked if they had the following symptoms in the last 2 weeks: (i) changes in sleep; (ii) changes in appetite; (iii) fatigue; (iv) feelings of sadness or irritability; (v) loss of interest in activities; (vi) inability to experience pleasure, feelings of guilt or worthlessness; (vii) inability to concentrate or making decisions; (viii) feeling restless or slowed down; and (ix) suicide thoughts. The response on each item had four categories ranging from $0=$ not at all to $3=$ nearly every day. Any "yes" response was defined as having depressive symptoms. The total score of PHQ-9 ranges from 0 to 27, with a score of 5 and more indicating depression. The Cronbach's alpha of PHQ-9 in the PINE study was $0.82[18,19]$.

Physical Function. We collect information on physical function of older adults by both self-report and physical performance testing. Self-reported physical function measures include Katz activities of daily living (ADL) [20], Lawton instrumental activities of daily living (IADL) [21], Index of Basic Physical Activities scale [22] and Index of Mobility scale [23]. For each self-reported physical function measures, the total score in each scale was used to present physical function. Higher scores of ADL, IADL, Index of Basic Physical Activities scale and Index of Mobility scale indicated higher levels of physical impairment. In this sample, ADL (Cronbach's alpha = 0.92), IADL (Cronbach's alpha $=0.90$ ), Index of Basic Physical Activities scale (Cronbach's alpha $=0.80$ ) and Index of Mobility scale (Cronbach's alpha = 0.80 ) had a good internal consistency.
With respect to physical performance testing, the Short Physical Performance Battery (SPPB) was used to collect information. Participants were asked to perform chair stand, tandem stand and timed walk. The SPPB has been validated among Chinese Americans and has good inter-rater reliability $[24,25]$. Higher scores of physical performance testing indicated higher levels of physical impairment.

Confounding Variables. Socio-demographic factors were controlled in data analysis, including age (in years), gender, education, annual income, marital status, living arrangement, number of children, years in the U.S. and medical comorbidities. Education was categorized into three groups: (i) elementary school and below; (ii) high school; and (iii) college and above. Selfreported annual income was divided into three groups: (i) $\$ 0$ $\$ 4,999$ per year; (ii) $\$ 5,000-\$ 9,999$ per year; and (iii) more than $\$ 10,000$ per year. Medical comorbidities were evaluated by the presence of nine diseases: (i) heart disease, heart attack, coronary thrombosis, coronary occlusion, or myocardial infarction; (ii) stroke or brain hemorrhage; (iii) cancer, malignancy, or a tumor of any type; (iv) high cholesterol; (v) diabetes, sugar in the urine, or high blood sugar; (vi) high blood pressure; (vii) a broken or fractured hip; (viii) thyroid disease; or (ix) osteoarthritis or inflammation or problems with joints [26-28].

\section{Data Analysis}

Chi-square and t-test were used to compare the differences in socio-demographics and physical function of older adults with depressive symptoms and without depressive symptoms. Chi-square and t-test were also applied to test the differences in socio-demographics and physical function of older adults with depression and without depression. Multivariate logistic regression models were employed to test the association between physical function and depressive symptoms/depression. Model A was adjusted for age and gender. Model B added education, income and marital status. Number of children, living arrangement and years in the U.S. were added in Model C. Model D added medical comorbidities to the previous model. In addition, all of the above models (Models A-D) were repeatedly using physical function with respect to depressive symptoms/depression outcomes. Odds ratios (ORs), 95\% confidence intervals (CIs), and significance levels were reported for multivariate analyses. All statistical analyses were conducted using SAS, Version 9.2 (SAS Institute Inc., Cary, North Carolina).

\section{Results}

The older adults in the study sample had a mean age of 72.8 years $(\mathrm{SD}=8.3$, range $=60-105)$ and $58.9 \%$ were female. The majority of participants (78.9\%) had equal or less than a high school education. Most of them (85.1\%) had an annual income less than US $\$ 10,000.71 .3 \%$ of participants were married, while $24.5 \%$ were widowed. More than half of the participants (55.6\%) averagely had 3 or more children. $21 \%$ of participants lived alone. $26.7 \%$ of the participants had been in the United States for less than 10 years. Details of the sample characteristics have been described elsewhere $[29,36]$. 


\section{PINE Study}

This study found self-reported physical function and performance-based physical function differed significantly by depressive symptoms (Table 1). Older adults with depressive symptoms were more likely to have higher scores in ADL (M: 0.29 vs. $0.08, \mathrm{p}<.001$ ), IADL (M: 2.24 vs. $1.18, \mathrm{p}<.001$ ), Index of Basic Physical Activities scale (M: 4.40 vs. 1.73, p <.001), Index of Mobility scale (M: 0.92 vs. $0.45, \mathrm{p}<.001)$, and SPPB (M: 5.30 vs. $3.87, \mathrm{p}<.001$ ) compared with those without depressive symptoms, illustrating that older adults with depressive symptoms are more likely to have poorer self-reported and performance-based physical function.

Similarly, self-reported physical function and performancebased physical function differed significantly by depression (Table 2). Older adults with depression were more likely to have higher scores in ADL (M: 0.51 vs. 0.13, p <.001), IADL (M: 3.21 vs. $1.46, \mathrm{p}<.001$ ), Index of Basic Physical Activities scale (M: 6.45 vs. $2.50, \mathrm{p}<.001)$, Index of Mobility scale (M: 1.36 vs. $0.58, \mathrm{p}<.001$ ), and SPPB (M: 6.45 vs. $4.28, p<.001$ ) than their counterparts without depression.

Table 3 showed ADL, IADL, Index of Mobility scale, Index of Basic Physical Activities scale and physical performance testing were significantly associated with depressive symptoms after controlling for age, gender, education, income, marital status, living arrangement, number of children, years in the U.S. and medical comorbidities. As for self-reported physical function, every one point higher in ADL impairment was associated with higher risk of depressive symptoms (OR: 1.29, 1.14-1.45). Greater levels of impairment in IADL were associated with higher risk of depressive symptoms (OR: 1.17, 1.13-1.22). Every one point higher in Index of Basic Physical Activities scale was associated with higher risk of depressive symptoms (OR: 1.22, 1.19-1.26). Older adults with higher scores in Index of Mobility scale were more likely to experience higher risk of depressive symptoms (OR: 1.52, 1.39-1.66). With regard to performancebased physical function, SPPB was significantly associated with depressive symptoms (OR: 1.16, 1.12-1.19) after controlling for all covariates. Specifically, every one point greater in tandem stand (OR: 1.33, 1.22-1.44), timed walk (OR: 1.23, 1.16-1.30) and chair stand (OR: 1.24, 1.18-1.31) was associated with higher risk of depressive symptoms.

In Table 4, we tested the association between physical function and depression. Consistently, both self-reported and performance-based physical function was significantly associated with depression. With respect to self-reported physical function and depression, every one point higher in ADL (OR: 1.32, 1.211.44), IADL (OR: 1.26, 1.21-1.31), Index of Basic Physical Activities scale (OR: 1.24, 1.21-1.28) and Index of Mobility scale (OR: 1.85, 1.69-2.04) was associated with higher risk of depression. As for performance-based physical function and depression, every one point higher in SPPB (OR: 1.23, 1.19-1.27) was associated with higher risk of depression. Every one point greater in tandem stand (OR: 1.35, 1.25-1.46), timed walk (OR: 1.41, 1.30-1.53) and chair stand (OR: 1.46, 1.36-1.57) was associated with higher risk of depression.
Table 1. Physical Function of PINE Study Participants with Any Depressive Symptoms

\begin{tabular}{|c|c|c|c|}
\hline & $\begin{array}{l}\text { Yes } \\
(\mathrm{n}=1423 ; \\
45.3 \%)\end{array}$ & $\begin{array}{l}\text { No } \\
(\mathrm{n}=1716 ; \\
54.67 \%)\end{array}$ & p-value \\
\hline \multicolumn{4}{|l|}{ Self-reported Physical Function } \\
\hline ADL & $0.29( \pm 1.09)$ & $0.08( \pm 0.57)$ & $<.001$ \\
\hline IADL & $2.24( \pm 2.87)$ & $1.18( \pm 2.07)$ & $<.001$ \\
\hline $\begin{array}{l}\text { Index of Basic Physical Activities } \\
\text { Scale }\end{array}$ & $4.40( \pm 4.44)$ & $1.73( \pm 2.87)$ & $<.001$ \\
\hline Index of Mobility Scale & $0.92( \pm 1.13)$ & $0.45( \pm 0.87)$ & $<.001$ \\
\hline
\end{tabular}

\section{Performance-based Physical Function}

\begin{tabular}{l|l|l|l} 
SPPB (reversely coded) & $5.30( \pm 3.51)$ & $3.87( \pm 2.73)<.001$
\end{tabular}

\begin{tabular}{|l|l|l|l} 
Tandem Stand (reversely coded) & $0.74( \pm 1.35)$ & $0.34( \pm 0.89)<.001$
\end{tabular}

\begin{tabular}{|l|l|l|l|l}
\hline Walk (reversely coded) & $2.28( \pm 1.45)$ & $1.83( \pm 1.42)<.001$
\end{tabular}

\begin{tabular}{|l|l|l|l|l}
\hline Chair (reversely coded) & $2.33( \pm 1.57)$ & $1.73( \pm 1.42)<.001$
\end{tabular}

Notes. Values are presented as n (\%) or mean \pm SD. ADL, IADL, Index of Basic Physical Activities scale, Index of Mobility scale, SPPB, Tandem stand, Walk and China were continuous variables. SPPB, Tandem stand, Walk and Chair were reversely coded, with higher scores indicating lower physical performance

Table 2. Physical Function of PINE Study Participants with Depression

\begin{tabular}{|c|c|c|c|}
\hline & Yes & No & p-value \\
\hline \multicolumn{4}{|l|}{ Self-reported Physical Function } \\
\hline ADL & $0.51( \pm 1.49)$ & $0.13( \pm 0.70)$ & $<.001$ \\
\hline IADL & $3.21( \pm 3.33)$ & $1.46( \pm 2.30)$ & $<.001$ \\
\hline $\begin{array}{l}\text { Index of Basic Physical Activities } \\
\text { Scale }\end{array}$ & $6.45( \pm 4.79)$ & $2.50( \pm 3.49)$ & $<.001$ \\
\hline Index of Mobility Scale & $1.36( \pm 1.22)$ & $0.58( \pm 0.96)$ & $<.001$ \\
\hline
\end{tabular}

\section{Performance-based Physical Function}

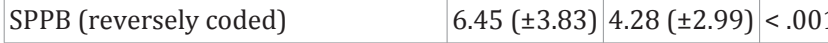

Tandem Stand (reversely coded) $\quad 1.05( \pm 1.61) 0.45( \pm 1.04)<.001$

\begin{tabular}{l|l|l}
\hline Walk (reversely coded) & $2.65( \pm 1.46)$ & $1.96( \pm 1.43)<.001$
\end{tabular}

\begin{tabular}{|l|l|l|l}
\hline Chair (reversely coded) & $2.83( \pm 1.62)$ & $1.89( \pm 1.46)<.001$
\end{tabular}

Notes. Values are presented as $n(\%)$ or mean \pm SD. ADL, IADL, Index of Basic Physical Activities scale, Index of Mobility scale, SPPB, Tandem stand, Walk and chair were continuous variables. SPPB, Tandem stand, Walk and Chair were reversely coded, with higher scores indicating lower physical performance.

\section{Discussion}

This study found both self-reported and directly observed physical function was significantly associated with depressive symptoms after controlling for age, gender, education, income, marital status, living arrangement, number of children, years in the U.S. and medical comorbidities. In addition, both self-reported and performance-based physical function was significantly associated with depression.

Our study found self-reported physical function was significantly associated with depressive symptoms. To be 
Association between both Self-Reported and Directly Observed Physical Function and Depressive Symptoms in a US Chinese Population: Findings from the PINE Study

\begin{tabular}{|c|c|c|c|c|}
\hline & Model A & Model B & Model C & Model D \\
\hline \multicolumn{5}{|c|}{ OR $(95 \% \mathrm{CI})$} \\
\hline Age & $1.02(1.01,1.02)^{*}$ & $1.02(1.01,1.03)^{*}$ & $1.02(1.01,1.03) \#$ & $1.02(1.00,1.03)^{*}$ \\
\hline Female & $1.52(1.31,1.75) \#$ & $1.52(1.30,1.78) \#$ & $1.52(1.30,1.79) \#$ & $1.41(1.20,1.66) \#$ \\
\hline Education & & $1.01(0.99,1.02)$ & $1.00(0.98,1.02)$ & $0.99(0.98,1.01)$ \\
\hline Income & & $0.85(0.80,0.91) \#$ & $0.86(0.80,0.93) \#$ & $0.87(0.81,0.93) \#$ \\
\hline Married & & $0.96(0.80,1.15)$ & $0.96(0.79,1.15)$ & $0.98(0.81,1.18)$ \\
\hline Living Arrangement & & & $1.00(0.96,1.04)$ & $1.01(0.97,1.05)$ \\
\hline Number of Children & & & $0.93(0.88,0.98)+$ & $0.93(0.88,0.98)^{*}$ \\
\hline Years in the U.S. & & & $1.00(0.99,1.00)$ & $1.00(0.99,1.00)$ \\
\hline Medical Comorbidities & & & & $1.24(1.17,1.31) \#$ \\
\hline ADL & $1.34(1.19,1.51) \#$ & $1.33(1.18,1.50) \#$ & $1.32(1.17,1.48) \#$ & $1.29(1.14,1.45) \#$ \\
\hline Age & $1.00(0.99,1.01)$ & $1.00(0.99,1.01)$ & $1.00(0.99,1.02)$ & $1.00(0.99,1.01)$ \\
\hline Female & $1.40(1.21,1.63) \#$ & $1.42(1.21,1.67) \#$ & $1.42(1.21,1.67) \#$ & $1.33(1.13,1.57) \#$ \\
\hline Education & & $1.02(1.00,1.03)$ & $1.01(0.99,1.02)$ & $1.00(0.98,1.02)$ \\
\hline Income & & $0.86(0.80,0.92) \#$ & $0.86(0.80,0.93) \#$ & $0.87(0.81,0.94) \#$ \\
\hline Married & & $0.93(0.77,1.13)$ & $0.93(0.76,1.12)$ & $0.94(0.77,1.14)$ \\
\hline Living Arrangement & & & $1.00(0.96,1.05)$ & $1.01(0.97,1.06)$ \\
\hline Number of Children & & & $0.92(0.86,0.97)+$ & $0.92(0.87,0.97)+$ \\
\hline Years in the U.S. & & & $1.00(0.99,1.00)$ & $0.99(0.99,1.00)$ \\
\hline Medical Comorbidities & & & & $1.21(1.14,1.28) \#$ \\
\hline IADL & $1.20(1.15,1.24) \#$ & $1.19(1.15,1.24) \#$ & $1.20(1.15,1.24) \#$ & $1.17(1.13,1.22) \#$ \\
\hline Age & $0.99(0.98,1.00)+$ & $0.99(0.98,1.00)^{*}$ & $0.99(0.98,1.01)$ & $0.99(0.98,1.00)$ \\
\hline Female & $1.18(1.01,1.38)^{*}$ & $1.23(1.04,1.45)^{*}$ & $1.24(1.05,1.46)^{*}$ & $1.20(1.01,1.42)^{*}$ \\
\hline Education & & $1.02(1.00,1.03)^{*}$ & $1.01(0.99,1.03)$ & $1.00(0.99,1.02)$ \\
\hline Income & & $0.88(0.82,0.94) \#$ & $0.89(0.82,0.95)^{*}$ & $0.89(0.82,0.96)^{*}$ \\
\hline Married & & $1.04(0.86,1.27)$ & $1.04(0.85,1.26)$ & $1.04(0.85,1.27)$ \\
\hline Living Arrangement & & & $1.01(0.96,1.05)$ & $1.01(0.97,1.06)$ \\
\hline Number of Children & & & $0.93(0.88,0.99)^{*}$ & $0.93(0.88,0.99)^{*}$ \\
\hline Years in the U.S. & & & $1.00(0.99,1.00)$ & $1.00(0.99,1.00)$ \\
\hline Medical Comorbidities & & & & 1.13(1.07,1.19)\# \\
\hline $\begin{array}{c}\text { Index of Basic Physical } \\
\text { Activities Scale }\end{array}$ & $1.24(1.21,1.28) \#$ & $1.22(1.19,1.26) \#$ & $1.24(1.21,1.27) \#$ & $1.22(1.19,1.26) \#$ \\
\hline Age & $1.00(0.99,1.01)$ & $1.00(0.99,1.01)$ & $1.01(0.99,1.02)$ & $1.00(0.99,1.01)$ \\
\hline Female & $1.41(1.21,1.63) \#$ & $1.43(1.22,1.68) \#$ & $1.43(1.22,1.68) \#$ & $1.35(1.15,1.59) \#$ \\
\hline Education & & $1.01(0.99,1.02)$ & $1.00(0.98,1.02)$ & $0.99(0.98,1.01)$ \\
\hline Income & & $0.85(0.79,0.91) \#$ & $0.86(0.80,0.93) \#$ & $0.86(0.80,0.93) \#$ \\
\hline Married & & $1.00(0.83,1.20)$ & $0.99(0.82,1.20)$ & $1.00(0.82,1.21)$ \\
\hline Living Arrangement & & & $1.01(0.97,1.06)$ & $1.02(0.98,1.06)$ \\
\hline Number of Children & & & $0.92(0.87,0.97)+$ & $0.92(0.87,0.98)+$ \\
\hline Years in the U.S. & & & $1.00(0.99,1.00)$ & $1.00(0.99,1.00)$ \\
\hline Medical Comorbidities & & & & $1.19(1.13,1.26) \#$ \\
\hline Index of Mobility Scale & $1.58(1.45,1.72) \#$ & 1.59 (1.46,1.73)\# & $1.59(1.46,1.73) \#$ & $1.52(1.39,1.66) \#$ \\
\hline Age & $0.99(0.98,1.00)$ & $0.99(0.98,1.00)$ & $1.00(0.99,1.01)$ & $1.00(0.99,1.01)$ \\
\hline Female & $1.38(1.19,1.60) \#$ & $1.44(1.22,1.69) \#$ & $1.44(1.22,1.69) \#$ & $1.36(1.16,1.61) \#$ \\
\hline Education & & $1.03(1.01,1.04)+$ & $1.01(1.00,1.03)$ & $1.01(0.99,1.03)$ \\
\hline Income & & $0.86(0.80,0.92) \#$ & $0.88(0.81,0.94) \#$ & $0.88(0.82,0.95) \#$ \\
\hline Married & & $0.99(0.82,1.20)$ & $0.98(0.81,1.19)$ & $1.00(0.82,1.21)$ \\
\hline Living Arrangement & & $1.17(1.14,1.21) \#$ & $1.01(0.97,1.05)$ & $1.02(0.97,1.06)$ \\
\hline Number of Children & & & $0.91(0.86,0.97)+$ & $0.91(0.86,0.97)^{*}$ \\
\hline Years in the U.S. & & & $0.99(0.99,1.00)$ & $0.99(0.99,1.00)^{*}$ \\
\hline Medical Comorbidities & & & & $1.18(1.12,1.25) \#$ \\
\hline SPPB & $1.16(1.13,1.19) \#$ & $1.17(1.14,1.21) \#$ & $1.18(1.14,1.21) \#$ & $1.16(1.12,1.19) \#$ \\
\hline
\end{tabular}


Association between both Self-Reported and Directly Observed Physical Function and Depressive Symptoms in a US Chinese Population: Findings from the PINE Study

\begin{tabular}{|c|c|c|c|c|}
\hline Age & $1.00(1.00,1.01)$ & $1.00(0.99,1.02)$ & $1.01(1.00,1.02)^{*}$ & $1.01(1.00,1.02)$ \\
\hline Female & $1.48(1.28,1.71) \#$ & $1.49(1.27,1.75) \#$ & $1.50(1.28,1.76) \#$ & $1.40(1.19,1.65) \#$ \\
\hline Education & & $1.01(1.00,1.03)$ & $1.01(0.99,1.02)$ & $1.00(0.98,1.02)$ \\
\hline Income & & $0.85(0.79,0.91) \#$ & $0.86(0.80,0.92) \#$ & $0.86(0.80,0.93) \#$ \\
\hline Married & & $0.95(0.79,1.15)$ & $0.95(0.78,1.14)$ & $0.96(0.80,1.17)$ \\
\hline Living Arrangement & & & $1.01(0.97,1.05)$ & $1.02(0.98,1.06)$ \\
\hline Number of Children & & & $0.93(0.88,0.98)+$ & $0.93(0.88,0.98)+$ \\
\hline Years in the U.S. & & & $1.00(0.99,1.00)$ & $0.99(0.99,1.00)$ \\
\hline Medical Comorbidities & & & & $1.21(1.15,1.28) \#$ \\
\hline Tandem Stand & $1.35(1.25,1.46) \#$ & 1.37 (1.26,1.48)\# & $1.37(1.27,1.49) \#$ & $1.33(1.22,1.44) \#$ \\
\hline Age & $1.01(1.00,1.02)$ & $1.01(1.00,1.02)$ & $1.02(1.01,1.03)^{*}$ & $1.01(1.00,1.02)^{*}$ \\
\hline Female & $1.47(1.27,1.70) \#$ & $1.51(1.29,1.77) \#$ & $1.51(1.29,1.77) \#$ & $1.41(1.20,1.65) \#$ \\
\hline Education & & $1.02(1.00,1.04)^{*}$ & $1.01(0.99,1.03)$ & $1.00(0.99,1.02)$ \\
\hline Income & & $0.85(0.80,0.91) \#$ & $0.87(0.81,0.94) \#$ & $0.88(0.82,0.94) \#$ \\
\hline Married & & $0.98(0.82,1.18)$ & $0.97(0.80,1.17)$ & $0.99(0.82,1.20)$ \\
\hline Living Arrangement & & & $1.01(0.97,1.05)$ & $1.02(0.98,1.06)$ \\
\hline Number of Children & & & $0.90(0.85,0.95) \#$ & $0.91(0.86,0.96) \#$ \\
\hline Years in the U.S. & & & $0.99(0.99,1.00)$ & $0.99(0.99,1.00)^{*}$ \\
\hline Medical Comorbidities & & & & $1.22(1.16,1.29) \#$ \\
\hline Walk & $1.21(1.15,1.28) \#$ & $1.23(1.16,1.30) \#$ & $1.26(1.19,1.33) \#$ & $1.23(1.16,1.30) \#$ \\
\hline Age & $1.00(0.99,1.01)$ & $1.00(0.99,1.01)$ & $1.01(1.00,1.02)^{*}$ & $1.01(1.00,1.02)$ \\
\hline Female & $1.42(1.23,1.64) \#$ & $1.44(1.22,1.69) \#$ & $1.44(1.23,1.69) \#$ & $1.36(1.16,1.60) \#$ \\
\hline Education & & $1.01(1.00,1.03)$ & $1.00(0.99,1.02)$ & $1.00(0.98,1.01)$ \\
\hline Income & & $0.87(0.81,0.93) \#$ & $0.88(0.81,0.94) \#$ & $0.88(0.82,0.95) \#$ \\
\hline Married & & $0.98(0.82,1.19)$ & $0.98(0.81,1.19)$ & $1.00(0.82,1.21)$ \\
\hline Living Arrangement & & & $1.00(0.96,1.05)$ & $1.01(0.97,1.05)$ \\
\hline Number of Children & & & $0.93(0.88,0.98)+$ & $0.93(0.88,0.98)^{*}$ \\
\hline Years in the U.S. & & & $1.00(0.99,1.00)$ & $0.99(0.99,1.00)$ \\
\hline Medical Comorbidities & & & & $1.20(1.13,1.27) \#$ \\
\hline Chair & $1.28(1.22,1.35) \#$ & $1.28(1.22,1.35) \#$ & $1.28(1.21,1.35) \#$ & $1.24(1.18,1.31) \#$ \\
\hline
\end{tabular}

Notes. Model A adjusted age and gender; Model B adjusted Model A + education, income, marital status; Model C adjusted Model B + living arrangement, number of children, years in the U.S.; Model D adjusted Model C + medical comorbidities.

${ }^{*} \mathrm{p}<0.05,{ }^{\mathrm{p}} \mathrm{p}<0.01,{ }^{\mathrm{p}} \mathrm{p}<0.001$

specific, the present study confirmed that US Chinese older adults with poorer report in ADL, IADL, Index of Basic Physical Activities scale and Index of Mobility scale were more likely to be associated with higher levels of depressive symptoms. Our results were consistent with early studies conducted in South Africa and Latin Americans that reported higher levels of impairment in self-reported physical function were associated with higher risk of depression $[8,9]$.

Our study goes beyond previous research by investigating the association between performance-based physical function and depressive symptoms. We found that US Chinese older adults with poorer performance in SPPB were more likely to be associated with higher risk of depressive symptoms. In addition, each item in SPPB (i.e. tandem stand, walk and chair) was significantly associated with depressive symptoms. Our study enables the comparison between both self-reported and directly observed physical function with depressive symptoms.

This study initially examined the association between both self-reported and performance-based physical function and depression. The results show higher scores in ADL, IADL, Index of Basic Physical Activities scale, Index of Mobility scale and SPPB were all significantly associated with higher levels of depression. This result indicates physical function impairment was not only associated with depressive symptoms, but also depression. Physical impairment is a risk factor for the psychological wellbeing of older adults.

These findings should be interpreted with cautions. First, we didn't use clinical diagnosis for depression. In our study, depression was measured by PHQ-9, with a score of 5 and more indicating depression. Second, although we tested the association between physical function and depressive symptoms, the reverse association may also exist. The mutual effects or causal effects were difficult to be proved in a cross-sectional study.

This study has significant research implications. First, the heterogeneity in measuring physical function has obfuscated the applicability and comparability of research findings. Our study provides insight into research on physical function and depressive symptoms by using different measures of selfreported and directly observed physical function. Second, this study also allows the comparison between physical function and both depressive symptoms and depression. 
Association between both Self-Reported and Directly Observed Physical Function and Depressive Symptoms in a US Chinese Population: Findings from the PINE Study

Table 4. Association between Physical Function and Depression

\begin{tabular}{|c|c|c|c|c|}
\hline & Model A & Model B & Model C & Model D \\
\hline \multicolumn{5}{|c|}{ OR (95\% CI) } \\
\hline Age & $1.01(1.00,1.03)^{*}$ & $1.01(1.00,1.02)$ & $1.01(0.99,1.03)$ & $1.01(0.99,1.02)$ \\
\hline Female & $1.48(1.22,1.80) \#$ & $1.45(1.17,1.79) \#$ & $1.44(1.16,1.78) \#$ & $1.35(1.08,1.68)+$ \\
\hline Education & & $1.02(1.00,1.04)$ & $1.01(0.99,1.03)$ & $1.01(0.99,1.03)$ \\
\hline Income & & $0.86(0.77,0.96)+$ & $0.86(0.77,0.96)+$ & $0.87(0.78,0.97) *$ \\
\hline Married & & $0.82(0.65,1.04)$ & $0.83(0.66,1.05)$ & $0.85(0.67,1.08)$ \\
\hline Living Arrangement & & & $0.97(0.92,1.03)$ & $0.97(0.91,1.04)$ \\
\hline Number of Children & & & $0.97(0.91,1.04)$ & $0.98(0.93,1.03)$ \\
\hline Years in the U.S. & & & $1.00(0.99,1.01)$ & $1.00(0.99,1.01)$ \\
\hline Medical Comorbidities & & & & $1.21(1.13,1.29) \#$ \\
\hline ADL & $1.34(1.23,1.47) \#$ & $1.35(1.23,1.47) \#$ & $1.34(1.23,1.47) \#$ & $1.32(1.21,1.44) \#$ \\
\hline Age & $0.98(0.97,1.00)+$ & $0.98(0.96,0.99)+$ & $0.98(0.97,1.00) *$ & $0.98(0.96,1.00)$ * \\
\hline Female & $1.34(1.09,1.65)+$ & $1.31(1.05,1.64)^{*}$ & $1.31(1.04,1.64)^{*}$ & $1.26(1.01,1.58)$ * \\
\hline Education & & $1.03(1.01,1.05)+$ & $1.02(1.00,1.04)$ & $1.02(0.99,1.04)$ \\
\hline Income & & $0.87(0.78,0.97)^{*}$ & $0.87(0.78,0.98)^{*}$ & $0.88(0.78,0.99) *$ \\
\hline Married & & $0.79(0.62,1.01)$ & $0.81(0.63,1.03)$ & $0.82(0.64,1.05)$ \\
\hline Living Arrangement & & & $0.97(0.91,1.03)$ & $0.97(0.92,1.03)$ \\
\hline Number of Children & & & $0.95(0.88,1.03)$ & $0.95(0.89,1.03)$ \\
\hline Years in the U.S. & & & $1.00(0.99,1.01)$ & $1.00(0.99,1.01)$ \\
\hline Medical Comorbidities & & & & $1.13(1.06,1.21) \#$ \\
\hline IADL & $1.27(1.22,1.32) \#$ & $1.27(1.23,1.33) \#$ & $1.28(1.23,1.33) \#$ & $1.26(1.21,1.31) \#$ \\
\hline Age & $0.98(0.97,0.99)+$ & $0.98(0.97,0.99)+$ & $0.98(0.96,0.99)+$ & $0.98(0.96,0.99)+$ \\
\hline Female & $1.06(0.86,1.31)$ & $1.09(0.86,1.37)$ & $1.09(0.86,1.37)$ & $1.07(0.85,1.35)$ \\
\hline Education & & $1.03(1.01,1.05)+$ & $1.03(1.01,1.05) *$ & $1.03(1.00,1.05)^{*}$ \\
\hline Income & & $0.91(0.81,1.01)$ & $0.90(0.81,1.02)$ & $0.91(0.81,1.02)$ \\
\hline Married & & & $0.89(0.69,1.15)$ & $0.90(0.70,1.15)$ \\
\hline Living Arrangement & & & $0.97(0.91,1.03)$ & $0.97(0.92,1.03)$ \\
\hline Number of Children & & & $0.99(0.92,1.06)$ & $0.99(0.92,1.06)$ \\
\hline Years in the U.S. & & & $1.00(0.99,1.01)$ & $1.00(0.99,1.01)$ \\
\hline Medical Comorbidities & & & & $1.06(0.98,1.13)$ \\
\hline $\begin{array}{c}\text { Index of Basic Physical Activities } \\
\text { Scale }\end{array}$ & $1.25(1.22,1.28) \#$ & $1.25(1.22,1.28) \#$ & $1.25(1.22,1.28) \#$ & $1.24(1.21,1.28) \#$ \\
\hline Age & $0.99(0.97,1.00)^{*}$ & $0.99(0.97,1.00)^{*}$ & $0.99(0.97,1.00)$ & $0.99(0.97,1.00)$ \\
\hline Female & $1.33(1.09,1.63)+$ & $1.31(1.05,1.63)^{*}$ & $1.30(1.04,1.63)^{*}$ & $1.26(1.00,1.57)^{*}$ \\
\hline Education & & $1.02(1.00,1.04)$ & $1.01(0.99,1.03)$ & $1.01(0.99,1.03)$ \\
\hline Income & & $0.86(0.77,0.96)+$ & $0.87(0.77,0.97) *$ & $0.87(0.78,0.98) *$ \\
\hline Married & & & $0.85(0.66,1.08)$ & $0.86(0.68,1.10)$ \\
\hline Living Arrangement & & & $1.00(0.94,1.06)$ & $1.00(0.95,1.06)$ \\
\hline Number of Children & & & $0.97(0.90,1.04)$ & $0.97(0.90,1.04)$ \\
\hline Years in the U.S. & & & $1.00(0.99,1.01)$ & $1.00(0.99,1.01)$ \\
\hline Medical Comorbidities & & & & $1.12(1.05,1.20) \#$ \\
\hline Index of Mobility Scale & $1.90(1.74,2.08) \#$ & $1.91(1.75,2.10) \#$ & $1.91(1.74,2.10) \#$ & $1.85(1.69,2.04) \#$ \\
\hline Age & $0.98(0.97,0.99)+$ & $0.98(0.96,0.99)+$ & $0.98(0.97,1.00)^{*}$ & $0.98(0.97,1.00) *$ \\
\hline Female & $1.29(1.05,1.58)^{*}$ & $1.30(1.04,1.63)^{*}$ & $1.29(1.03,1.62)^{*}$ & $1.25(0.99,1.56)$ \\
\hline Education & & $1.04(1.02,1.06) \#$ & $1.04(1.01,1.06)+$ & $1.03(1.01,1.05)+$ \\
\hline Income & & $0.87(0.77,0.97)+$ & $0.89(0.79,1.00) *$ & $0.89(0.79,1.00)$ * \\
\hline Married & & $0.81(0.64,1.04)$ & $0.81(0.64,1.04)$ & $0.83(0.65,1.05)$ \\
\hline Living Arrangement & & & $0.99(0.93,1.04)$ & $0.99(0.94,1.05)$ \\
\hline Number of Children & & & $0.97(0.90,1.04)$ & $0.97(0.90,1.04)$ \\
\hline Years in the U.S. & & & $0.99(0.99,1.00)$ & $1.00(0.98,1.00)$ \\
\hline Medical Comorbidities & & & & $1.13(1.05,1.21) \#$ \\
\hline SPPB & $1.23(1.19,1.27) \#$ & $1.24(1.20,1.29) \#$ & $1.24(1.20,1.29) \#$ & $1.23(1.19,1.27) \#$ \\
\hline
\end{tabular}


Association between both Self-Reported and Directly Observed Physical Function and Depressive Symptoms in a US Chinese Population: Findings from the PINE Study

\begin{tabular}{|c|c|c|c|c|}
\hline Age & $1.00(0.99,1.01)$ & $1.00(0.99,1.01)$ & $1.00(0.99,1.02)$ & $1.00(0.98,1.01)$ \\
\hline Female & $1.42(1.16,1.74) \#$ & $1.38(1.11,1.72)+$ & $1.38(1.11,1.71)+$ & $1.30(1.05,1.63)$ * \\
\hline Education & & $1.02(1.00,1.05)^{*}$ & $1.02(1.00,1.04)$ & $1.02(0.99,1.04)$ \\
\hline Income & & $0.86(0.77,0.95)+$ & $0.86(0.77,0.97) *$ & $0.87(0.78,0.98)$ * \\
\hline Married & & $0.78(0.62,0.99)^{*}$ & $0.79(0.62,1.00)^{*}$ & $0.81(0.63,1.03)$ \\
\hline Living Arrangement & & & $0.99(0.93,1.04)$ & $0.99(0.94,1.05)$ \\
\hline Number of Children & & & $0.98(0.92,1.06)$ & $0.98(0.92,1.06)$ \\
\hline Years in the U.S. & & & $1.00(0.99,1.01)$ & $1.00(0.99,1.01)$ \\
\hline Medical Comorbidities & & & & $1.18(1.10,1.26) \#$ \\
\hline Tandem Stand & $1.38(1.28,1.48) \#$ & $1.39(1.29,1.50) \#$ & $1.39(1.29,1.50) \#$ & $1.35(1.25,1.46) \#$ \\
\hline Age & $1.00(0.99,1.02)$ & $1.00(0.99,1.02)$ & $1.01(0.99,1.02)$ & $1.00(0.99,1.02)$ \\
\hline Female & $1.40(1.15,1.70) \#$ & $1.42(1.15,1.77)+$ & $1.41(1 \cdot 13,1.75)+$ & $1.33(1.06,1.65)^{*}$ \\
\hline Education & & $1.04(1.02,1.06) \#$ & $1.03(1.01,1.05)+$ & $1.02(1.00,1.05)$ * \\
\hline Income & & $0.85(0.67,1.07)+$ & $0.88(0.78,0.99) *$ & $0.88(0.79,0.99)$ * \\
\hline Married & & & $0.85(0.67,1.07)$ & $0.86(0.68,1.09)$ \\
\hline Living Arrangement & & & $0.99(0.93,1.04)$ & $0.99(0.94,1.05)$ \\
\hline Number of Children & & & $0.94(0.87,1.01)$ & $0.94(0.87,1.01)$ \\
\hline Years in the U.S. & & & $0.99(0.98,1.00)$ & $0.99(0.98,1.00)$ \\
\hline Medical Comorbidities & & & & $1.18(1.11,1.26) \#$ \\
\hline Walk & $1.38(1.28,1.48) \#$ & $1.42(1.31,1.53) \#$ & $1.44(1.33,1.56) \#$ & $1.41(1.30,1.53) \#$ \\
\hline Age & $1.00(0.98,1.01)$ & $0.99(0.98,1.01)$ & $0.99(0.98,1.01)$ & $0.99(0.98,1.01)$ \\
\hline Female & $1.34(1.09,1.64)+$ & $1.31(1.05,1.63)^{*}$ & $1.30(1.04,1.62)^{*}$ & $1.25(0.99,1.56)$ \\
\hline Education & & $1.02(1.00,1.04)$ & $1.01(0.99,1.04)$ & $1.01(0.99,1.03)$ \\
\hline Income & & $0.89(0.80,0.99)^{*}$ & $0.90(0.80,1.00) *$ & $0.90(0.80,1.01)$ \\
\hline Married & & $0.82(0.65,1.05)$ & $0.83(0.65,1.06)$ & $0.84(0.66,1.07)$ \\
\hline Living Arrangement & & & $0.98(0.93,1.04)$ & $0.98(0.93,1.04)$ \\
\hline Number of Children & & & $0.98(0.91,1.06)$ & $0.98(0.91,1.06)$ \\
\hline Years in the U.S. & & & $1.00(0.99,1.01)$ & $1.00(0.99,1.01)$ \\
\hline Medical Comorbidities & & & & $1.14(1.06,1.22) \#$ \\
\hline Chair & $1.49(1.39,1.60) \#$ & $1.50(1.40,1.61) \#$ & $1.50(1.40,1.60) \#$ & $1.46(1.36,1.57) \#$ \\
\hline
\end{tabular}

Notes. Model A adjusted age and gender; Model B adjusted Model A + education, income, marital status; Model C adjusted Model B + living arrangement, number of children, years in the U.S.; Model D adjusted Model C + medical comorbidities.

${ }^{*} \mathrm{p}<0.05,{ }^{\mathrm{p}} \mathrm{p}<0.01,{ }^{*} \mathrm{p}<0.001$

In policy practice, our study suggests health professionals should be aware of the depressive symptoms or depression in older adults with physical function impairment. We found various kinds of physical impairment were significantly associated with depressive symptoms and depression. Poorer report in ADL, IADL, Index of Basic Physical Activities scale, Index of Mobility scale and poorer performance in SPPB were associated with higher risk of depressive symptoms and depression. Health professionals are suggested to pay more attention on older adults with physical impairment and conduct preventions for them when needed.

In future research, the causal effects of self-reported and performance-based physical function on depressive symptoms may be strengthened by longitudinal research. Studies on testing the combined effects of self-reported and performance-based physical function on depressive symptoms are expected. Future research can also examine the association between cognitive impairment and depressive symptoms.

\section{Conclusion}

This study shows both self-reported and directly observed physical function were significantly associated with depressive symptoms after controlling for age, gender, education, income, marital status, living arrangement, number of children, years in the U.S. and medical comorbidities. The result for the association between physical function and depression is consistent with the association between physical function and depressive symptoms. Future research may focus on the causal effects of self-reported and performance-based physical function on depressive symptoms.

\section{References}

1. Aneshensel CS, Frerichs RR, Huba GJ. Depression and physical illness: A multiwave, nonrecursive causal model. Journal of Health and Social Behavior. 1984; 25(4):350-371.

2. Ormel J, Rijsdijk FV, Sullivan M, van Sonderen E, Kempen GIJM. Temporal and reciprocal relationship between IADL/ADL disability and depressive symptoms in late life. The Journals of Gerontology Series B: Psychological Sciences and Social Sciences. 2002;57(4):P338-P347.

3. Rodríguez López S, Montero P, Carmenate M, Avendano M. Functional decline over 2 years in older Spanish adults: evidence from the Survey of Health, Ageing and Retirement in Europe. Geriatrics \& gerontology international. 2014;14(2):403-412. doi: 10.1111/ggi.12115.

4. Penninx BWJH, Deeg DJH, van Eijk JTM, Beekman ATF, Guralnik 


\section{PINE Study}

JM. Changes in depression and physical decline in older adults: a longitudinal perspective. Journal of affective disorders. 2000;61(12):1-12.

5. Dalle Carbonare L, Maggi S, Noale M, Giannini S, Rozzini R, Cascio VL, et al. Physical disability and depressive symptomatology in an elderly population: a complex relationship. The Italian Longitudinal Study on Aging (ILSA). The American Journal of Geriatric Psychiatry. 2009;17(2):144-154

6. Weinberger MI, Raue PJ, Meyers BS, Bruce ML. Predictors of new onset depression in medically ill, disabled older adults at 1 year follow-up. The American journal of geriatric psychiatry. 2009;17(9):802-809.

7. Gayman MD, Turner RJ, Cui M. Physical limitations and depressive symptoms: exploring the nature of the association. The Journals of Gerontology Series B: Psychological Sciences and Social Sciences. 2008;63(4):S219-S228

8. Tomita A, Burns JK. Depression, disability and functional status among community-dwelling older adults in South Africa: evidence from the first South African National Income Dynamics Study. Internationa journal of geriatric psychiatry. 2013;28(12):1270-1279. doi: 10.1002/ gps.3954.

9. Guerra M, Ferri CP, Sosa AL, Salas A, Gaona C, Gonzales V, et al. Latelife depression in Peru, Mexico and Venezuela: the 10/66 populationbased study. The British Journal of Psychiatry. 2009;195(6):510 515. doi: 10.1192/bjp.bp.109.064055

10. Dong X, Wong E, Simon MA. Study design and implementation of the PINE study. Journal of aging and health. 2014: 26(7):1085-99. doi $10.1177 / 0898264314526620$

11. Dong X. The population study of Chinese elderly in Chicago. Journal of aging and health. 2014;26(7):1079-1084.

12. Dong X, Chang ES, Simon M, Wong E. Sustaining Community-University Partnerships: Lessons learned from a participatory research project with elderly Chinese. Gateways: International Journal of Community Research and Engagement. 2011;4:31-47.

13. Dong X, Chang ES, Wong E, Simon M. Working with culture: lessons learned from a community-engaged project in a Chinese aging population. Aging Health. 2011;7(4):529-537. DOI 10.2217/ahe.11.43

14. Dong X, Li Y, Chen R, Chang ES, Simon M. Evaluation of community health education workshops among Chinese older adults in Chicago: A community-based participatory research approach. Journal of Education and Training Studies. 2013;1(1):p170-181.

15. Matthew Magee MPH, Shah AM, Guo L, Cheung W, Dong X, Simon MA. Building a Chinese community health survey in Chicago: the value of involving the community to more accurately portray health. 2008

16. Simon MA, Chang ES, Rajan KB, Welch MJ, Dong X. Demographic characteristics of US Chinese older adults in the Greater Chicago area assessing the representativeness of the PINE study. Journal of aging and health. 2014;26(7):1100-1115. doi: 10.1177/0898264314543472.

17. American Psychiatric A. Diagnostic and statistical manual of mental disorders American Psychiatric Association. Washington, DC. $1994 ; 210$.

18. Dong X, Chen R, Li C, Simon MA. Understanding depressive symptoms among community-dwelling Chinese older adults in the greater Chicago area. Journal of aging and health. 2014;26(7):1155-1171. doi: 10.1177/0898264314527611.

19. Chang ES, Beck T, Simon MA, Dong X. A psychometric assessment of the psychological and social well-being indicators in the PINE study. Journal of aging and health. 2014;26(7):1116-1136. doi: $10.1177 / 0898264314543471$.

20. Katz S, Akpom CA. A measure of primary sociobiological functions. International journal of health services. 1976;6(3):493-508.
21. Lawton MP, Brody EM. Assessment of older people: self-maintaining and instrumental activities of daily living. Nursing Research. 1970;19(3): 278.

22. Nagi SZ. An epidemiology of disability among adults in the United States. The Milbank Memorial Fund Quarterly Health and Society. 1976: 54(4): 439-467. DOI: $10.2307 / 3349677$.

23. Rosow I, Breslau N. A Guttman health scale for the aged. Journal of gerontology. 1966;21(4):556-559.

24. Dong X, Simon M, Evans D. Decline in Physical Function and Risk of Elder Abuse Reported to Social Services in a Community-Dwelling Population of Older Adults. Journal of the American Geriatrics Society. 2012;60(10):1922-1928. doi: 10.1111/j.1532-5415.2012.04147.

25. Dong X, Simon M, Fulmer T, de Leon CFM, Rajan B, Evans DA. Physical function decline and the risk of elder self-neglect in a communitydwelling population. The Gerontologist. 2009:gnp164.

26. Dong X, Chen R, Wu B, Zhang NJ, Mui A-S, Chi I. Association between Elder Mistreatment and Suicidal Ideation among Community-Dwelling Chinese Older Adults in the USA. Gerontology. 2015;62(1):71-80. doi: $10.1159 / 000437420$.

27. Simon MA, Chen R, Chang ES, Dong X. The Association Between Filial Piety and Suicidal Ideation: Findings From a Community-Dwelling Chinese Aging Population. The Journals of Gerontology Series A: Biological Sciences and Medical Sciences. 2014;69(Suppl 2):S90-S97. doi: 10.1093/gerona/glu142

28. Dong X, Chen R, Simon MA. The prevalence of medical conditions among US Chinese community-dwelling older adults. The Journals of Gerontology Series A: Biological Sciences and Medical Sciences. 2014;69(Suppl 2):S15-S22. doi: 10.1093/gerona/glu151

29. Chen R, Simon MA, Chang ES, Zhen Y, Dong X. The Perception of Social Support Among US Chinese Older Adults Findings From the PINE Study. Journal of aging and health. 2014;26(7):1137-1154. doi: 10.1177/0898264314529332.

30. Dong X, Zhang M, Simon MA. The expectation and perceived receipt of filial piety among Chinese older adults in the Greater Chicago area. Journal of aging and health. 2014;26(7):1225-1247. doi: $10.1177 / 0898264314541697$

31. Dong $X$, Chang ES. Social Networks among the Older Chinese Population in the USA: Findings from the PINE Study. Gerontology 2017.

32. Dong X, Li M. Self-reported and directly observed physical function and anxiety symptoms in community-dwelling US Chinese older adults: Findings from the PINE study. J Psychol Cognition 2016;1:2936.

33. Dong X, Bergren SM. The Associations and Correlations Between Self-reported Health and Neighborhood Cohesion and Disorder in a Community-dwelling U.S. Chinese Population. The Gerontologist 2016:gnw050.

34. Dong X. Sociodemographic and socioeconomic characteristics of elder self-neglect in an US Chinese aging population. Archives of gerontology and geriatrics 2016;64:82-9.

35. Dong X, Simon M. Prevalence of elder self-neglect in a Chicago Chinese population: The role of cognitive physical and mental health. Geriatrics \& gerontology international 2015.

36. Dong XQ, Li G. Caregiver Abuse of Chicago Chinese Older Adults in a Community-Dwelling Population. Journal of geriatric medicine and gerontology 2015;1. 